\title{
Gaia Space Mission and Quasars
}

\author{
Tomaž Zwitter* \\ Faculty of Mathematics and Physics, University of Ljubljana, Ljubljana, Slovenia
}

\section{OPEN ACCESS}

Edited by:

Paola Marziani,

Osservatorio Astronomico di Padova

(INAF), Italy

Reviewed by:

Maurizio Paolillo,

Università degli Studi di Napoli

Federico II, Italy

Mary Loli Martínez-Aldama,

Instituto de Astrofísica de Andalucía

(CSIC), Spain

*Correspondence:

Tomaž Zwitter

tomaz.zwitter@fmf.uni-lj.si

Specialty section:

This article was submitted to

Milky Way and Galaxies,

a section of the journal

Frontiers in Astronomy and Space

Sciences

Received: 28 August 2017 Accepted: 27 October 2017 Published: 15 November 2017

Citation:

Zwitter T (2017) Gaia Space Mission and Quasars.

Front. Astron. Space Sci. 4:41. doi: 10.3389/fspas.2017.00041
Quasars are often considered to be point-like objects. This is largely true and allows for an excellent alignment of the optical positional reference frame of the ongoing ESA mission Gaia with the International Celestial Reference Frame. But presence of optical jets in quasars can cause shifts of the optical photo-centers at levels detectable by Gaia. Similarly, motion of emitting blobs in the jet can be detected as proper motion shifts. Gaia's measurements of spectral energy distribution for around a million distant quasars is useful to determine their redshifts and to assess their variability on timescales from hours to years. Spatial resolution of Gaia allows to build a complete magnitude limited sample of strongly lensed quasars. The mission had its first public data release in September 2016 and is scheduled to have the next and much more comprehensive one in April 2018. Here we briefly review the capabilities and current results of the mission. Gaia's unique contributions to the studies of quasars are already being published, a highlight being a discovery of a number of quasars with optical jets.

Keywords: extragalactic astronomy, active galactic nuclei, quasars, quasar spectral energy distribution, extragalactic radio jets, strong gravitational lensing, astrometry, photometry

\section{THE GAIA MISSION}

Gaia, an ongoing mission of ESA (gaia.esa.int), is conducting a massive all sky optical survey of sources with $0.0 \leq V \leq 20.9$ (for current review of mission properties and its status see Prusti et al., 2016). Brown et al. (2016) published an initial sky chart with 1,142,679,969 sources, which already makes Gaia the largest all-sky survey of celestial objects to date. Gaia is primarily an astrometric mission, positional accuracy at the end of the mission is expected to be between 5 and $400 \mu$ as (Figure 1), while the spatial resolution is $\sim 0.1$ arc-second (as). This impressive accuracy of position of optical photo-center is mostly photon-noise limited and in many cases supersedes the accuracy of VLBI positions for radio emitting sources. Gaia is continuously scanning the skies. In 5 years of official mission lifetime it will observe each source in the sky transiting its focal plane from 50 to over 200 times, median is 72 . Sampling is uneven, with the shortest separations corresponding to two observations within the 6-h spin period of the satellite. Gaia includes three main instruments: astrometry is done in white light ( $\mathrm{G}$ band, between 330 and 1,050 nm), photometry is collected via weakly dispersed spectra in the blue (BP, blue photometer, 330-680 nm) and red (RP, red photometer, $640-1,050 \mathrm{~nm}$ ) bands, each sampled in wavelength by 45 pixels. $76 \%$ of energy of a sharp line from a point source is spread in the wavelength direction over $\sim 1.6$ pixels $(\mathrm{BP})$ and $\sim 3.8$ pixels (RP). Finally there is a spectroscopic instrument on-board which is collecting spectra in the $845-872 \mathrm{~nm}$ range for objects brighter than $V \sim 15.5$ and at a resolving power of 10,500. The main goal of spectroscopic instrument is to determine radial velocities, but for the very bright stars $(V \leq 12)$ it will also measure abundances of chemical elements with lines in wavelength range of the spectrograph. Magnitude and wavelength limitations of Gaia's spectroscopic instrument are being complemented by ground-based optical stellar spectroscopic surveys which are aimed to obtain stellar parameters and accurate radial velocities, as well as abundances of individual elements for 


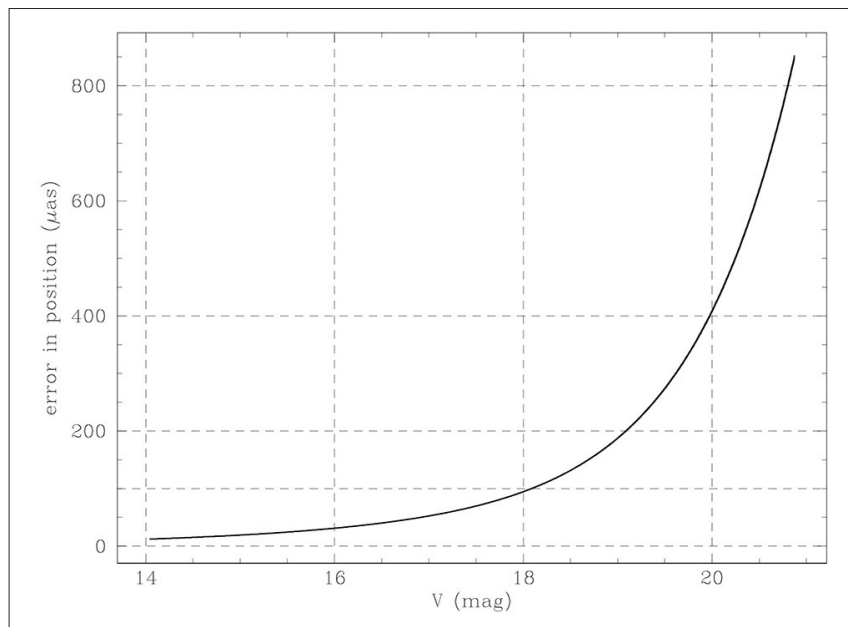

FIGURE 1 | Positional error after a 5-year mission as a function of $\checkmark$ magnitude. The latter was derived from Gaia's intrinsic $G$ magnitude using the relation $V=1.02 G-0.24$, which is appropriate for quasars with $z \leq 4$ (Proft et al., 2015). Positional error estimate assumes a point-like source and follows Equations (4-7) from Prusti et al. (2016) with $0.54<V-I<0.98$.

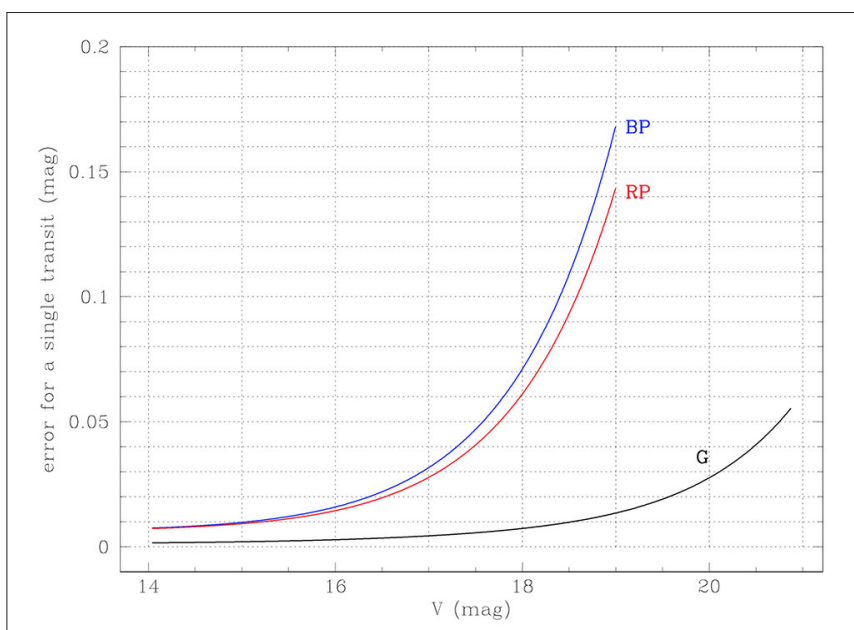

FIGURE 2 | Errors in integrated G (white light), RP (red photometer), and BP (blue photometer) magnitudes per transit, as a function of $V$ magnitude, estimated from $\mathrm{G}$ magnitude as explained in Figure 1. Errors were estimated from Equations (9-13) in Prusti et al. (2016) using $N_{o b s}=1$. Combining blocks of $N_{\text {obs }}$ transits obtained on timescales of days or weeks reduces the errors by $\sqrt{N_{\text {obs }}}$. stars which are not within the reach of Gaia. They can however observe only a small fraction of such sources. The ongoing surveys include RAVE (Kunder et al., 2017, https://www.ravesurvey.org/project/), LAMOST (Cui et al., 2012, http://www. lamost.org), Gaia-ESO (Gilmore et al., 2012, https://www.gaiaeso.eu/), and Galah (https://galah-survey.org, de Silva et al., 2015, see also Martell et al. 2017).

\section{GAIA AND QUASARS}

A vast majority of quasars are fainter than $V \sim 15.5$ so they are not observed by the spectroscopic instrument aboard Gaia, but the mission's astrometry and spectrophotometric BP and RP measurements are extremely relevant for quasar studies. Figure 1 shows that one may expect a positional accuracy of $\sim 70 \mu$ as at $V \sim 17$ and $\sim 400 \mu$ as at $V \sim 20$ at the end of the official 5-year mission lifetime. These figures may actually be too pessimistic, as Gaia performs better than expected on the faint end, and the mission itself may be extended up to 2022 or beyond, therefore increasing the photon budget and time span of its observations. Figure 2 shows accuracy of integrated magnitudes over the $G, \mathrm{BP}$, and RP bands for each transit. It demonstrates the potential of Gaia's $\sim 72$ observations of each quasar over a $\sim 5$ year timeframe to assess temporal variability for a complete population of quasars down to $V \sim 20$ in white light ( $G$ band). For bright sources $(V<18)$ one can also study color changes on timescales from hours to years, using their integrated BP and RP magnitudes.

$\mathrm{BP}$ and RP instruments do not collect only integrated magnitudes but also spectrophotometric information. Figure 3 shows a typical quasar spectrum (black tracing), and its BP (blue) and RP (red) spectrophotometry collected by Gaia over a 5-year mission. The panels show the signal for objects with apparent magnitude $V=20$ which have 4 different redshifts between 0.5 and 3.5. Vertical stripe at $\sim 8,600 \AA$ is the wavelength range of Gaia's spectroscopic instrument. In our view the figure, which summarizes simulations of Proft et al. (2015), clearly demonstrates the ability of Gaia to determine photometric redshifts for a complete population of quasars down to $V \sim 20$. For brighter objects even changes in their spectral energy distribution (SED) should be detectable. Proft et al. (2015) demonstrate that variability of SDSS $154757.71+060626.6$, which has Sloan red magnitude $r^{\prime}=17.5$ and redshift $z=2.03$, is clearly within the reach of Gaia.

Gaia mission observes any object bright enough to trigger its instruments. So the initial quasar catalog with 1,248,372 sources, which was prepared before the launch by Andrei et al. (2014), is only a lower limit of what we may expect to be observed. Properties of the catalog are summarized in Table 1. Note the relatively low astrometry precision for most of the quasars. Position is of course more accurately known for 4,866 VLBI sources which are part of the catalog and for additional 38,699 sources with available radio position, although of lower precision.

\section{QUASARS IN GAIA'S FIRST DATA RELEASE}

Gaia's first data release (Gaia DR1) was published in September 2016 (Brown et al., 2016). It contains

1. Positions, parallaxes (error $\sim 0.3$ milli arc-second, hereafter mas) and proper motions (error $\sim 1$ mas/yr) for 2 million Hipparcos and Tycho stars;

2. Positions (error $\sim 10$ mas) and $G$ magnitudes (error $<0.03 \mathrm{mag}$ ) for 1.1 billion objects $(V \leq 20.9)$;

3. G-band light curves and characterization for $\sim 3,000$ Cepheids and RR Lyrae stars around the south ecliptic pole. 

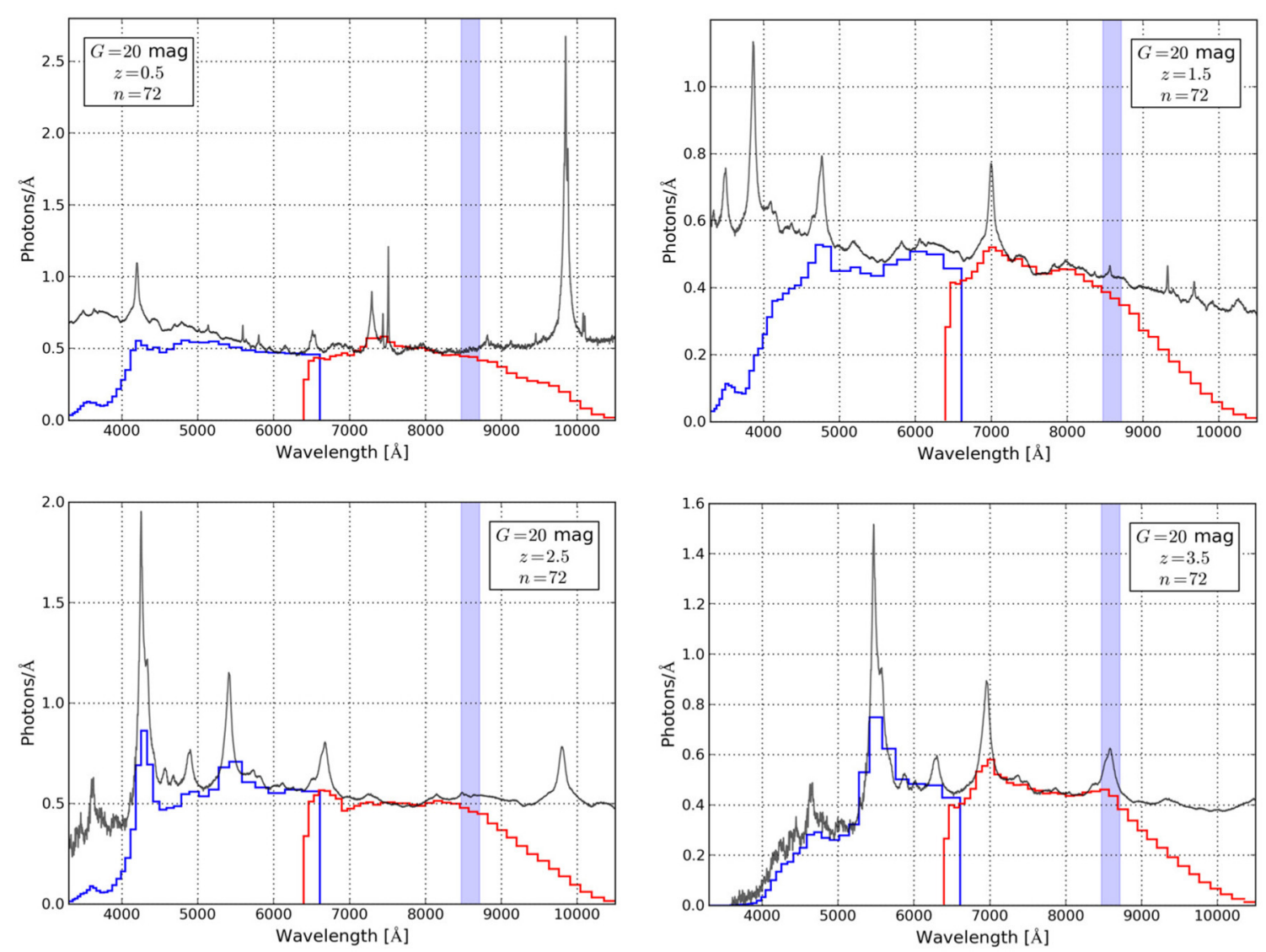

FIGURE 3 | Ability of Gaia to determine redshifts of quasars with $G=20$ (corresponding to $V \approx 20.2$ ) after a median of 72 exposures collected over a 5 -year mission. From Proft et al. (2015), with permission.

TABLE 1 | Properties of the Initial quasar catalog with numbers of different sources and their expected typical precision, adapted from Andrei et al. (2014).
Number of sources

Sources with magnitude

Sources with redshift

Sources with $G<20$

Sources with $G>21$

Sources with $z<1.0$

Sources with $z>2.0$

Astrometry precision (arc sec)

Magnitude precision

Redshift precision

Average density (sources/deg ${ }^{2}$ )

Average neighbor distance (arc sec)
$1,248,372$

$1,246,512$

$1,157,285$

371,098

690,507

250,405

383,487

1

0.5

0.01

30.3

220
Quasars observed within the first data release have been discussed by Lindegren et al. (2016) and Mignard et al. (2016). Main results can be summarized as follows: Some 135,000 quasars from the list of Andrei et al. (2014) were included in the astrometric solution, and their positions were determined with an (inflated) standard uncertainty of 1 mas. Accurately known positions from VLBI were used to align the Gaia DR1 reference frame with the extragalactic radio frame. A special astrometric solution has been computed for selected extragalactic objects with optical counterparts in Gaia DR1, including the ones from the second realization of the International Celestial Reference Frame (ICRF2). Formal standard error for 2,191 such quasars (with $17.6<V<20.7$ ) does not exceed 0.76 mas for $50 \%$ and 3.35 mas for $90 \%$ of the sources. Alignment of the Gaia DR1 reference frame with the ICRF2 is better than 0.1 mas at epoch 2015.0. The two frames do not rotate to within 0.03 mas/yr. There are now 11,444 objects with VLBI positions, i.e., 3.5-times more than in ICRF2 (Petrov and Kovalev, 2017).

\section{OFFSETS BETWEEN GAIA AND VLBI POSITIONS AND EVIDENCE FOR OPTICAL JETS}

Lindegren et al. (2016) note that for sources with good optical and radio astrometry they found no indication of physical optical vs. radio offsets exceeding a few tens of mas, in most cases they are less than 1 mas. This in encouraging, as it will permit to build a very accurate common Gaia+radio reference frame in the 

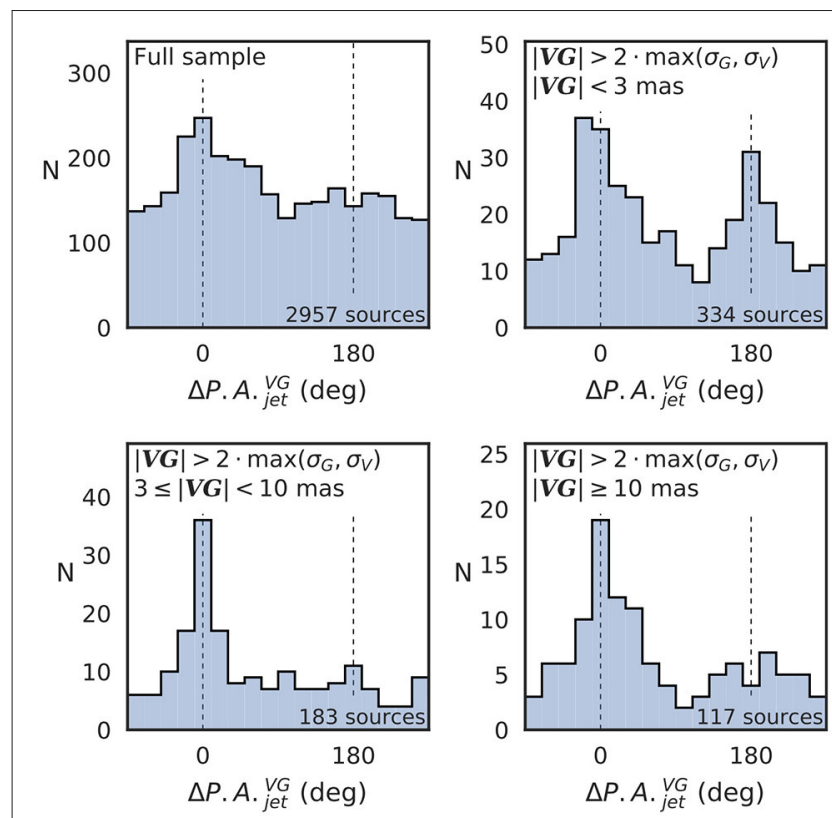

FIGURE 4 | Histograms of differences in position angle ( $\triangle P . A$.$) between the$ direction of the radio jet (jet) and the direction of the offset $(V G)$ from radio to optical position of the source. All but the top-left panel plot only objects with the size of the offset $V G$ being at least two times larger than the errors of their VLBI $\left(\sigma_{V}\right)$ and Gaia $\left(\sigma_{G}\right)$ positions. Vertical dashed lines indicate aligned and counter-aligned jets. From Kovalev et al. (2017), with permission.

future. But some objects may require further checks. In particular, Makarov et al. (2017) publish a list of 188 objects (out of 2,293 ICRF2 sources), most of them with $z<0.5$, with offsets up to 1 arcsec, the latter corresponds to a distance of $\sim 7.5 \mathrm{kpc}$ at $z \sim 0.5$. They propose that 89 of them may be AGNs (quasars and BL Lac) dislodged from their host galaxies' centers.

In a much larger study Petrov and Kovalev (2017) used the whole 1.1 billion object dataset from Gaia DR1 and the VLBI absolute astrometry catalog RFC 2016c to find 6,055 secure matches with AGNs. Kovalev et al. (2017) used this large sample to check on offsets between radio and optical positions. In 2,957 AGNs they were able to determine the direction of the radio jet. They find a significant prevalence of optical and radio offsets in directions along or opposite the one of the radio jet (Figure 4). This suggests that strong, extended optical jet structures are present in many AGN. Position offsets along the jet require strong, extended parsec-scale optical jets, while small ( $<1$ mas) offsets in direction opposite to the jet can be due to extended VLBI jet structure or a "core-shift" effect due to synchrotron opacity.

\section{CONCLUSIONS}

Gaia is living to its promise of revolutionizing virtually any field of astronomy. In case of quasars two of the instruments on board are of particular interest: spectrophotometric $\mathrm{BP} / \mathrm{RP}$ instrument and astrometric instrument. The former allows studies of SED variability for a complete sample of sources with $V<18$, and of general brightness variations and photometric redshift determination for all sources brighter than $V \sim 20$. The emphasis is on completeness, and on the ability to discover and characterize yet unknown quasars in this magnitude range. Both present a major advance to current lively research efforts to characterize quasar variability (e.g., Peters et al., 2016; Marziani et al., 2017; Rumbaugh et al., 2017). An obvious importance of astrometry of quasars is to harmonize optical and radio reference frames. Some 135,000 quasars, which were already analyzed within the first data release, already allowed to achieve an unprecedented accuracy of alignment of VLBI radio and optical Gaia reference frames. A small offset between optical and radio position, which is seen in some quasars with well determined directions of radio jets, points to presence of strong, extended optical jets on parsec scales. Spatial resolution of Gaia allows to discover large numbers of gravitationally lensed quasars (Lemon et al., 2017), especially when photometric and spectrophotometric measurements of Gaia are combined with mid-infrared selection, as enabled by the WISE mission (Agnello, 2017).

Gaia observations are ongoing, with the second public data release planned for April 2018. It will include a fiveparameter astrometric solutions for all sources with acceptable formal standard errors ( $>10^{9}$ are anticipated), and positions (R.A., Dec.) for sources for which parallaxes and proper motions cannot be derived. Next, it will include $G$ and integrated BP and RP photometric magnitudes for all sources. For sources brighter than $G_{R V S}=12$ also median radial velocities will be published. Finally for stars brighter than $G$ $=17$ there will be estimates of the effective temperature and, where possible, line-of-sight extinction, based on the above photometric data. Of less interest to quasar studies, a list of photometric data for a sample of variable stars and epoch astrometry for a pre-selected list of over 10,000 asteroids will be provided.

There are further planned intermediate public data releases, with the final database to be published some 3 years after the end of observations, which may happen anytime between 2019 and 2023. By then a complete magnitude limited sample of quasars, many of them new, together with their variability, redshift and in many cases optical jets or multiple gravitationally lensed images are bound to be discovered.

\section{AUTHOR CONTRIBUTIONS}

The author confirms being the sole contributor of this work and approved it for publication.

\section{ACKNOWLEDGMENTS}

The author acknowledges financial support of the Slovenian Research Agency (research core funding No. P1-0188). This research has been partially funded by the European Space Agency contract 4000111918. 


\section{REFERENCES}

Agnello, A. (2017). Quasar lenses and galactic streams: outlier selection and Gaia multiplet detection. Month. Notices R. Astron. Soc. 471, 2013-2021. doi: $10.1093 / \mathrm{mnras} / \mathrm{stx} 1650$

Andrei, A. H., Antón, S., Taris, F., Bourda, G., Souchay, J., Bouquillon, J., et al. (2014). "The gaia initial quasar catalog," in Proceedings of the Journées 2013: "Systèmes de référence spatio-temporels," ed N. Capitaine, 84-87.

Brown, A. G. A., Vallenari, A., Prusti, T., de Bruijne, J. H. J., Mignard, F., Drimmel, R., et al. (2016). Gaia data release 1. Summary of the astrometric, photometric, and survey properties. Astron. Astrophys. 595:23. doi: 10.1051/0004-6361/201629512

Cui, X. Q., Zhao, Y. H., Chu, Y. Q., Li, G. P., Li, Q., Zhang, L. P., et al. (2012). The large sky area multi-object fiber spectroscopic telescope (LAMOST). Res. Astron. Astrophys. 12, 1197-1242. doi: 10.1088/1674-4527/12/9/003

De Silva, G. M., Freeman, K. C., Bland-Hawthorn, J., Martell, S., de Boer, E. W., Asplund, M., et al. (2015). The GALAH survey: scientific motivation. Month. Notices R. Astron. Soc. 449, 2604-2617. doi: 10.1093/mnras/stv327

Gilmore, G., Randich, S., Asplund, M., Binney, J., Bonifacio, P., Drew, J., et al. (2012). The Gaia-ESO public spectroscopic survey. Messenger 147, 25-31.

Kovalev, Y. Y., Petrov, L. and Plavin, A. V. (2017). VLBI-Gaia offsets favor parsec-scale jet direction in active galactic nuclei. Astron. Astrophys. 598:4. doi: 10.1051/0004-6361/201630031

Kunder, A., Kordopatis, G., Steinmetz, M., Zwitter, T., McMillan, P. J., Casagrande, L., et al. (2017). The radial velocity experiment (RAVE): fifth data release. Astron. J. 153:30. doi: 10.3847/1538-3881/153/2/75

Lemon, C. A., Auger, M. W., McMahon, R. G., and Koposov, S. E. (2017). Gravitationally lensed quasars in gaia: I. Resolving small-separation lenses. arXiv:1709.08976.

Lindegren, L., Lammers, U., Bastian, U., Hernández, J., Klioner, S., Hobbs, D., et al. (2016). Gaia data release 1. Astrometry: one billion positions, two million proper motions and parallaxes. Astron. Astrophys. 595:32. doi: 10.1051/0004-6361/201628714

Makarov, V. V., Frouard, J., Berghea, C. T., Rest, A., Chambers, K. C., Kaiser, N., et al. (2017). Astrometric evidence for a population of dislodged AGNs. Astrophys. J. Lett. 835:6. doi: 10.3847/2041-8213/835/2/L30
Martell, S. L., Sharma, S., Buder, S., Duong, L., Schlesinger, K. J., Simpson, J., et al. (2017). The GALAH survey: observational overview and Gaia DR1 companion. Month. Notices R. Astron. Soc. 465:3203. doi: 10.1093/mnras/s tw2835

Marziani, P., Bon, E., Grieco, A., Bon, N., Dultzin, D., Del Olmo, A., et al. (2017). "Optical variability patterns of radio-quiet and radio-loud quasars," in New Frontiers in Black Hole Astrophysics, Proceedings of the IAU Symposium, Vol. 324, 243-244.

Mignard, F., Klioner, S., Lindegren, L., Bastian, U., Bombrun, A., Hernández, J., et al. (2016). Gaia data release 1. Reference frame and optical properties of ICRF sources. Astron. Astrophys. 595:16. doi: 10.1051/0004-6361/2016 29534

Peters, C. M., Richards, G. T., Myers, A. D., Strauss, M. A., Schmidt, K. B., Ivezić, Ž., et al. (2016). Quasar classification using color and variability. Astrophys. J. 811:29.

Petrov, L., and Kovalev, Y. Y. (2017). On significance of VLBI/Gaia position offsets. Month. Notices R. Astron. Soc. 467, L71-L75. doi: 10.1093/mnrasl/s $1 \mathrm{x} 001$

Proft, S., and Wambsganss, J. (2015). Exploration of quasars with the Gaia mission. Astron. Astrophys. 574:11. doi: 10.1051/0004-6361/201323280

Prusti, T., de Bruijne, J. H. J., Brown, A. G. A., Vallenari, A., Babusiaux, C., Bailer-Jones, C. A. L., et al. (2016). The Gaia mission. Astron. Astrophys. 595:36. Rumbaugh, N., Shen, Y., Morganson, E., Liu, X., Banerji, M., McMahon, R. G., et al. (2017). Extreme variability quasars from the Sloan Digital Sky Survey and the Dark Energy Survey. arXiv:1706.07875.

Conflict of Interest Statement: The author declares that the research was conducted in the absence of any commercial or financial relationships that could be construed as a potential conflict of interest.

Copyright (c) 2017 Zwitter. This is an open-access article distributed under the terms of the Creative Commons Attribution License (CC BY). The use, distribution or reproduction in other forums is permitted, provided the original author(s) or licensor are credited and that the original publication in this journal is cited, in accordance with accepted academic practice. No use, distribution or reproduction is permitted which does not comply with these terms. 